\title{
Ex-Press $\AA^{\circledR}$ Surgery Versus Trabeculectomy for Primary Open Angle Glaucoma with Low Preoperative Intraocular Pressure
}

\section{Mitsuya Otsuka}

University of Toyama: Toyama Daigaku

Atsushi Hayashi

University of Toyama: Toyama Daigaku

Naoki Tojo ( $\square$ tojo-naoki@umin.ac.jp )

University of Toyama https://orcid.org/0000-0002-8036-4781

\section{Research Article}

Keywords: Ex-Press, glaucoma, low IOP, NTG, trabeculectomy

Posted Date: January 14th, 2022

DOI: https://doi.org/10.21203/rs.3.rs-1243065/v1

License: (c) (i) This work is licensed under a Creative Commons Attribution 4.0 International License.

Read Full License 


\section{Abstract}

Purpose: To compare surgical outcomes between Ex-PRESS ${ }^{\circledR}$ surgery (EXP) and trabeculectomy (Trab) for primary open angle glaucoma (POAG) with low preoperative intraocular pressure (IOP).

Patients and Methods: This was a retrospective non-randomized study. We included POAG patients with preoperative IOP $\leq 16 \mathrm{mmHg}$ who were taking tolerance glaucoma medications. We compared the surgical outcomes, postoperative IOP, number of glaucoma medications, reduction rate of corneal endothelial cell density (ECD), visual acuity, and postoperative complications between POAG patients who underwent EXP (34 eyes) or Trab (38 eyes) and could be followed for $>2$ years.

Results: Both surgeries significantly decreased the IOP ( $\mathrm{p}<0.001)$ : at 2 years, EXP provided decreases from $13.4 \pm 2.3$ to $10.2 \pm 3.1 \mathrm{mmHg}$, Trab provided decreases from $13.5 \pm 2.0$ to $8.9 \pm 3.2 \mathrm{mmHg}$. No significant differences were observed in the postoperative IOP $(p=0.076)$, number of postoperative medications $(p=0.263)$, success rate $(p=0.900)$, reduction rate of ECD $(p=0.410)$, or difference in visual acuity $(p=0.174)$. The reduction rate of IOP was significantly high in the Trab group $(p=0.047)$.

Conclusions: Both surgeries significantly decreased IOP and were useful surgical methods for low-IOP glaucoma. Our results suggest that trabeculectomy can decrease IOP more than Ex-PRESS surgery but might have more complications.

\section{Introduction}

Various surgical methods for glaucoma have been created since the 2000s. For example, minimally invasive glaucoma surgery (MIGS) and tube shunt surgeries are performed worldwide. Many MIGS result in intraocular pressure (IOP) that is in the $\mathrm{mmHg}$ range of mid-teen values [1], and it might be difficult to achieve postoperative IOP at values lower than the mid-teens. Tube shunt surgery is difficult to perform for patients with low preoperative IOP, due to the risk of hypotony [2]. For these reasons, there are limited surgical options for glaucoma patients with low preoperative IOP.

Patients with progressive visual field impairment despite low IOP are more common in Japan [3].

Trabeculectomy (Trab) is the most common glaucoma surgery for patients with low IOP. In a Trab, a bleb is formed in the sub-tenon space to receive the outflow of aqueous humor, thereby lowering the IOP. In addition, in cases in which a Trab is performed, the postoperative IOP can be adjusted with suture-lysis, suturing, or needling. In patients with low preoperative IOP in particular, strict IOP control is required after a Trab is conducted. Trabeculectomy was reported to be effective for low IOP glaucoma, with an improvement in the deterioration of the visual field [4-9].

With another surgical method, i.e., Ex-PRESS ${ }^{8}$ (Alcon Laboratories, Fort Worth, TX) (EXP) surgery, the postoperative IOP can also be adjusted [10]. The EXP is a stainless-steel filtration device designed to shunt the aqueous humor from the anterior chamber to the sub-tenon space. EXP has some similarities to Trab, but there are some important differences between these two types of surgery. With the EXP, 
trabecular meshwork and the peripheral iris do not have to be excised. The EXP is also thought to be less invasive than a Trab. Generally, the EXP has the merits of low risks of hypotonic maculopathy, choroidal detachment, and shallow anterior chamber because the amount of aqueous humor exiting the bleb from the anterior chamber is limited [11]. However, due to the limited outflow of aqueous humor, it is unclear whether the IOP can be sufficiently reduced for a long term [12]. There are few reports of surgical outcomes of EXP for low-IOP glaucoma [13-15].

Many investigations have compared the surgical outcomes of Trab and EXP, and several reports state that these surgeries' ability to lower IOP is comparable [16-18]. We have found no reports of comparisons of these surgeries for low-IOP glaucoma patients. In the present study, we compared the surgical outcomes between EXP and Trab surgery for patients with low preoperative IOP. We evaluated seven factors: (1) the postoperative IOP, (2) the number of postoperative glaucoma medications, (3) the rate of the reduction of IOP from the preoperative IOP, (4) the postoperative surgical success rate, (5) the reduction rate of corneal endothelial cell density (ECD), (6) visual acuity (VA), and (7) postoperative complications.

\section{Patients And Methods}

\section{Patients}

This was a retrospective, non-randomized observational study. Seventy-two patients (76 eyes) underwent glaucoma surgeries. Four patients underwent surgery for both eyes; we used the unilateral data of the eye that was operated earlier. We analyzed the cases of a final total of 72 consecutive patients who underwent EXP (EXP group: 34 eyes) surgery or Trab (Trab group: 38 eyes) for the first time at Toyama University Hospital and were followed for $>2$ years. We performed these surgeries for patients with primary open angle glaucoma (POAG) and the mean preoperative IOP $\leq 16 \mathrm{mmHg}$. We defined the preoperative IOP as the mean IOP of the patient's three visits just before he or she underwent preoperative treatment.

All cases during the period from September 2014 to January 2020 were recruited. We performed EXP from September 2014 to March 2018, and after that we performed Trab in all cases. We included patients who simultaneously underwent cataract surgery. Glaucoma patients other than those with POAG and who had undergone other glaucoma surgery were also excluded. Two glaucoma specialists (N.T. and A.H.) diagnosed the cases of POAG.

All patients underwent a comprehensive ophthalmic examination including refraction, Goldmann gonioscopy, Goldmann applanation tonometry (GAT), a fundus examination, automated perimetry (Humphrey Field Analyzer; Carl Zeiss Meditec, Dublin, CA), measurement with optical coherent tomography (OCT) RS-3000 (Nidek; Aichi, Japan), the measurement of central corneal ECD with an EM4000 specular microscope (Tomey; Nagoya, Japan), and the measurement of the central corneal 
thickness (CCT) with AS-OCT (CASIA SS-1000; Tomey, Nagoya, Japan). The IOP was measured by GAT. We did not fix the time point for the measurement of IOP.

The patients had already used maximally tolerated glaucoma medications but required further treatment to lower their IOP due to the progression of their visual-field disorder. The surgical indication was judged by one glaucoma specialist (N.T.). The research protocol was approved by the Institutional Review Board of the University of Toyama, and the procedures used conformed to the tenets of the Declaration of Helsinki. After the nature and possible consequences of the study were explained to the patients, written informed consent was obtained from all individual participants included in the study.

\section{Surgical techniques}

All patients were operated on by one surgeon (N.T.), who has abundant experience performing EXP and Trab. The EXP surgical technique in all cases was as follows. Retrobulbar anesthesia was administered. A standard fornix-based conjunctival incision was made to gain exposure to the scleral bed adjacent to the limbus. A single $3.5-\mathrm{mm}^{2}$ square scleral flap was created. Mitomycin C (MMC) solution $(0.04 \mathrm{mg} / \mathrm{ml})$ was applied below the conjunctiva and below the scleral flap for $4 \mathrm{~min}$. At this point, the eye was a completely enclosed space, and thus the MMC solution could not flow into the anterior chamber. The treated area was then irrigated with approx. $100 \mathrm{ml}$ of balanced salt solution. If the patient needed simultaneous cataract surgery, the cataract surgery was performed at this time. Phacoemulsification was performed with a WhiteStar Signature system (Abbott Medical Optics, Santa Ana, CA), and an intraocular lens (IOL) was implanted from the clear temporal cornea. Regarding the surgical indications for cataract surgery, since the present study was a retrospective analysis, no clear criteria were established for visual acuity (VA), the Emery grade, or patient age; cataract surgery was performed based on the operator's judgment.

The scleral flap was lifted, and a 25-ga. needle was horizontally inserted into the anterior chamber at the surgical limbus to create a path for the Ex-PRESS ${ }^{\circledR}$ (model P50); the 25-ga. needle was inserted into the anterior chamber from the sclera-cornea transition zone parallel with the iris. The Ex-PRESS shunt was then inserted into the anterior chamber. The scleral flap was sutured using 10-0 nylon while the tension on the sutures was adjusted to maintain the anterior chamber depth with a slow flow of aqueous humor around the margins of the scleral flap. Most of the cases were sutured with two stitches. The conjunctiva was meticulously closed with 10-0 nylon sutures. We confirmed that there was no leakage from the blebs.

The Trab surgical technique was as follows, in all cases. The differences from the EXP were that: (1) a 4$\mathrm{mm}^{2}$ square scleral flap consisting of a double layer was created; (2) the trabecular meshwork was excised; and (3) the peripheral iris was incised. Most of the cases were sutured with four stitches.

\section{Postoperative medication}

The postoperative treatment protocol was the same in both the EXP and Trab groups. The postoperative treatments consisted of topical steroids, antibiotics, and non-steroidal anti-inflammatory drugs (NSAIDs). The antibiotics were applied for 4-6 weeks after the surgery. The steroid and NSAIDs were reduced over a 
12-week period after the interventions. After the surgeries, glaucoma medications were stopped in all cases. Glaucoma medications were added at the discretion of the patients' physicians. We counted a compounded agent as two medications.

\section{Evaluation of the factors}

We used two success criteria. We defined a successful surgery as a post-operative reduction in the IOP $>20 \%$ (Criterion A) or $>30 \%$ (Criterion B) from the preoperative IOP. We did not use the absolute value of postoperative IOP for the definition of success. We defined failure as meeting one of the following conditions: (1) post-operative IOP reduced $<20 \%$ or $30 \%$ from the preoperative IOP on two consecutive visits after the first postoperative month; (2) postoperative IOP $<5 \mathrm{mmHg}$ on two consecutive visits after the first postoperative month; (3) requiring additional glaucoma surgery; or (4) phthisis or loss of light perception. The definition of success did not include the use or non-use of glaucoma medications.

We examined the ECD at the center of the cornea with the EM-4000 specular microscope, which automatically calculates the density value. The measured values were obtained only once before the surgery and at 1 and 2 years after the surgery. We defined the reduction rate of ECD as the ratio of postoperative ECD from pre-operative ECD. Since cataract surgery is known to affect ECD, we compared the survival rate in ECD, excluding cases that underwent simultaneous cataract surgery.

We measured the patients' VA with decimal visual acuity. We converted the values to the logMAR (minimum angle of resolution) and evaluated the change of VA. Since cataract surgery is known to affect postoperative VA, we compared the difference of preoperative VA and postoperative VA, excluding cases that underwent simultaneous cataract surgery.

We evaluated the postoperative complications as (1) shallow anterior chamber (subjectivity observed with a slit lamp), choroidal detachment, hypotony maculopathy (fold in macula due to hypotony), hyphema (niveau formation), vitreous hemorrhage, vitreous prolapse, leaking bleb (needed suture or needling), visual acuity aggravation (aggravated more than 0.2 with logMAR), and bullous keratopathy (the ECD could not be measured with the EM-4000 microscope).

\section{Statistical analysis}

A Wilcoxon signed-rank test and Student's t-test were used. A log-rank test was used for the comparison of the results of a Kaplan-Meier analysis. All of the statistical analyses were performed with JMP Pro 14 software (SAS, Cary, NC). Assuming that the standard deviation of the postoperative IOP was $3.0 \mathrm{mmHg}$, we found that a total of 34 pairs of values was necessary to detect a meaningful difference of $2.0 \mathrm{mmHg}$ with respect to the IOP daily variation with $80 \%$ power and the two-sided significance level of 0.05 . Significance was defined as p-values $<0.05$.

\section{Results}

\section{Ophthalmic data}


We analyzed 72 patients. The surgeries were conducted without intraoperative complications such as expulsive hemorrhage. All cases were followed-up without additional glaucoma surgery within 2 years. The characteristics of the EXP and Trab groups are summarized in Table 1. No ophthalmic parameters were significantly different between the two groups.

\begin{tabular}{|c|c|c|c|}
\hline \multicolumn{4}{|c|}{$\begin{array}{c}\text { Table } 1 \\
\text { Patients' characteristics }\end{array}$} \\
\hline & $\begin{array}{l}\text { Ex-press } \\
\text { (34eyes) }\end{array}$ & $\begin{array}{l}\text { Trab } \\
\text { (38 eyes) }\end{array}$ & p-value \\
\hline Age, yrs & $70.1 \pm 12.2$ & $68.4 \pm 10.0$ & 0.519 \\
\hline Simultaneous cataract surgery, eyes & $11 / 34(32.3 \%)$ & $17 / 38(44.7 \%)$ & 0.242 \\
\hline CCT, mm & $523 \pm 33$ & $518 \pm 28$ & 0.527 \\
\hline Preoperative IOP, mmHg & $13.4 \pm 2.3$ & $13.5 \pm 2.0$ & 0.860 \\
\hline Preoperative medications & $3.9 \pm 0.6$ & $3.5 \pm 0.9$ & 0.145 \\
\hline Preoperative ECD, cells $/ \mathrm{mm}^{2}$ & $2294 \pm 441$ & $2350 \pm 362$ & 0.677 \\
\hline Preoperative visual acuity, logMAR & $0.402 \pm 0.376$ & $0.222 \pm 0.378$ & 0.051 \\
\hline ECD: endothelial corneal cells density & & & \\
\hline
\end{tabular}

The postoperative IOP data are summarized in Table 2. The means of the postoperative IOPs at 1, 3, 6 and 18 months were significantly higher in the EXP group compared to the Trab group. The postoperative medications are summarized in Table 3. In the EXP group, the mean numbers of glaucoma medications used at 6 and 12 months were significantly higher than those in the Trab group. The EXP patients thus tended to require more glaucoma medications. The reduction rate of IOP is described in Table 4. At 2 years post-surgery, the IOP reduction rate in the EXP group was a $22.8 \%$ decrease, and that in the Trab group was a $33.4 \%$ decrease. The mean of the reduction rate of IOP was significantly higher in the Trab group $(p=0.0471)$. 
Table 2

Comparison of postoperative IOP between Ex-press and trabeculectomy

\begin{tabular}{|llll|}
\hline & Ex-press & Trab & p-value \\
\hline preoperative & $13.4 \pm 2.3$ & $13.5 \pm 2.0$ & 0.860 \\
\hline $1 \mathrm{mo}$ & $8.8 \pm 4.8$ & $6.4 \pm 3.5$ & 0.0142 \\
\hline $2 \mathrm{mos}$ & $9.4 \pm 4.0$ & $7.5 \pm 3.9$ & 0.0514 \\
\hline $3 \mathrm{mos}$ & $9.6 \pm 3.7$ & $7.6 \pm 3.0$ & 0.0104 \\
\hline $6 \mathrm{mos}$ & $9.1 \pm 2.2$ & $7.6 \pm 3.2$ & 0.0277 \\
\hline $12 \mathrm{mos}$ & $9.5 \pm 2.8$ & $8.3 \pm 3.3$ & 0.0841 \\
\hline $18 \mathrm{mos}$ & $10.0 \pm 2.2$ & $8.6 \pm 3.2$ & 0.0353 \\
\hline $24 \mathrm{mos}$ & $10.2 \pm 3.1$ & $8.9 \pm 3.2$ & 0.0758 \\
\hline Data are $\mathrm{mmHg}$. & & \\
\hline
\end{tabular}

Table 3

The number of postoperative glaucoma medications

\begin{tabular}{|llll|}
\hline & Ex-press & Trab & p-value \\
\hline Preoperative & $3.9 \pm 0.6$ & $3.7 \pm 0.8$ & 0.145 \\
\hline 3 mos & $0.3 \pm 1.0$ & $0.2 \pm 0.6$ & 0.459 \\
\hline 6 mos & $0.9 \pm 1.4$ & $0.5 \pm 1.2$ & 0.221 \\
\hline 12 mos & $1.4 \pm 1.7$ & $0.6 \pm 1.3$ & 0.024 \\
\hline 18 mohs & $1.8 \pm 1.9$ & $1.2 \pm 1.5$ & 0.140 \\
\hline 24 mos & $1.9 \pm 1.8$ & $1.4 \pm 1.6$ & 0.263 \\
\hline
\end{tabular}


Table 4

Rate of IOP decline

\begin{tabular}{|llll|}
\hline & Ex-press & Trabe & p-value \\
\hline 3 mos & $27.0 \pm 27.5$ & $44.1 \pm 21.3$ & 0.0042 \\
\hline $6 \mathrm{mos}$ & $30.4 \pm 20.1$ & $43.0 \pm 23.9$ & 0.0188 \\
\hline $12 \mathrm{mos}$ & $28.0 \pm 20.6$ & $37.8 \pm 25.6$ & 0.0825 \\
\hline $18 \mathrm{mos}$ & $23.0 \pm 23.2$ & $35.6 \pm 25.1$ & 0.0349 \\
\hline $24 \mathrm{mos}$ & $22.8 \pm 21.1$ & $33.4 \pm 24.2$ & 0.0471 \\
\hline \multicolumn{4}{|l}{ The data are percentages. IOP: intraocular pressure. } \\
\hline
\end{tabular}

\section{Success rate}

The results of the Kaplan-Meier analysis are illustrated in Figures 1 and 2. The surgical outcomes were not significantly different (Criterion $A, p=0.900$; Criterion $B, p=0.150$; log-rank test). The respective success rates for Criterion $A$ at $6,12,18$, and 24 months were $85.3 \%, 79.4 \%, 76.5 \%$, and $76.5 \%$ in the EXP group and $94.7 \%, 84.2 \%, 79.0 \%$, and $76.3 \%$ in the Trab group. The respective success rates for Criterion $B$ at 6 , 12,18 , and 24 months were $67.5 \%, 55.9 \%, 44.1 \%$ and $38.2 \%$ in the EXP group and $76.3 \%, 68.4 \%, 63.2 \%$, and $52.6 \%$ in the Trab group.

We defined success without medications as complete success. The ratio of complete success (Criterion A) was $32.4 \%$ (11 eyes) in EXP group and $42.1 \%$ (16 eyes) in Trab group, there was no significant difference $(p=0.393)$. The ratio of complete success (Criterion B) was $17.6 \%$ ( 6 eyes) in EXP group and $31.6 \%$ (12 eyes) in Trab group, there was no significant difference $(p=0.173)$.

\section{The survival rate of ECD}

The mean ECD value and survival rate of ECD are shown in Table 5. The survival rate of ECD after 2 years was not significantly different between the EXP $(94.8 \%)$ and Trab $(92.3 \%)$ groups $(p=0.410)$. Since cataract surgery is a factor that affects ECD, we also show data excluding cases of simultaneous cataract surgery. The survival rate of ECD after 2 years was not significantly different between the EXP (92.6\%) and Trab (90.5\%) groups ( $\mathrm{p}=0.731)$. 
Table 5

Corneal endothelial cell density and survival rate

\begin{tabular}{|llll|}
\hline total & $\begin{array}{l}\text { Ex-press } \\
(\mathbf{3 4} \text { eye) }\end{array}$ & $\begin{array}{l}\text { Trab } \\
\text { (38 eyes) }\end{array}$ & p-value \\
\hline Preoperative ECD, cells/mm² & $2294 \pm 441$ & $2350 \pm 362$ & 0.677 \\
\hline 12 mos ECD, cells $/ \mathrm{mm}^{2}$ & $2194 \pm 565$ & $2305 \pm 471$ & 0.470 \\
\hline 24 mos ECD, cells/mm ${ }^{2}$ & $2182 \pm 502$ & $2178 \pm 477$ & 0.663 \\
\hline 12 mos Survival rate, \% & $95.4 \pm 14.2$ & $98.3 \pm 14.2$ & 0.629 \\
\hline 24 mos Survival rate, \% & $94.8 \pm 14.1$ & $92.3 \pm 20.8$ & 0.410 \\
\hline Excluding the cases of simultaneous cataract surgery & Ex-press & Trab & \\
\hline Preoperative ECD, cells/mm ${ }^{2}$ & $(\mathbf{2 3 ~ e y e )}$ & $(\mathbf{2 1}$ eyes) & \\
\hline 12 mos ECD, cells/mm ${ }^{2}$ & $2247 \pm 507$ & $2309 \pm 422$ & 0.662 \\
\hline 24 mos ECD, cells/mm ${ }^{2}$ & $2077 \pm 532$ & $2292 \pm 501$ & 0.185 \\
\hline 12 mos Survival rate, \% & $2117 \pm 640$ & $2098 \pm 497$ & 0.917 \\
\hline 24 mos Survival rate, \% & $92.6 \pm 12.1$ & $99.7 \pm 15.1$ & 0.104 \\
\hline ECD: endothelial corneal cells density & $92.6 \pm 13.4$ & $90.5 \pm 25.0$ & 0.731 \\
\hline
\end{tabular}

\section{Best Collected Visual acuity}

Table 6 provides the data of the mean VA and the difference from the preoperative VA to the postoperative VA. Since cataract surgery is a factor that affects VA, we also show data excluding cases of simultaneous cataract surgery. In the EXP group, there were many cases of poor preoperative VA, but there was no significant difference versus the Trab group $(p=0.051)$. Regarding the difference in VA before and after surgery, the VA had declined at 1 year and 2 years post-surgery in the Trab group, whereas there was no decline at 1 and 2 years post-surgery in the EXP group. The patients who underwent the EXP procedure tended to have less visual loss than those who underwent the Trab procedure, but the difference was not significant $(p=0.469)$. Since cataract surgery is a factor that affects VA, we also show data excluding cases of simultaneous cataract surgery. The results were similar, visual loss after EXP was less, but no significant difference $(p=0.251)$. 
Table 6

Changes in visual acuity (VA)

\begin{tabular}{|llll|}
\hline total & $\begin{array}{l}\text { Ex-press } \\
(\mathbf{2 3} \text { eyes })\end{array}$ & $\begin{array}{l}\text { Trab } \\
(\mathbf{2 1} \text { eyes })\end{array}$ & p-value \\
\hline Preoperative VA (logMAR) & $0.402 \pm 0.376$ & $0.222 \pm 0.373$ & 0.051 \\
\hline 12 mos (logMAR) & $0.461 \pm 0.421$ & $0.320 \pm 0.420$ & 0.158 \\
\hline 24 mos (logMAR) & $0.469 \pm 0.451$ & $0.390 \pm 0.565$ & 0.562 \\
\hline 12 mos VA difference & $0.059 \pm 0.192$ & $0.097 \pm 0.246$ & 0.471 \\
\hline 24 mos VA difference & $0.067 \pm 0.228$ & $0.176 \pm 0.409$ & 0.174 \\
\hline Excluding the case of simultaneous cataract surgery & Ex-press & Trab & p-value \\
\hline Preoperative VA (logMAR) & $(23$ eyes) & $(21$ eyes) & \\
\hline 12 mos (logMAR) & $0.420 \pm 0.375$ & $0.253 \pm 0.419$ & 0.170 \\
\hline 24 mos (logMAR) & $0.504 \pm 0.379$ & $0.332 \pm 0.495$ & 0.200 \\
\hline 12 mos VA difference & $0.508 \pm 0.403$ & $0.506 \pm 0.668$ & 0.988 \\
\hline 24 mos VA difference & $0.085 \pm 0.177$ & $0.079 \pm 0.240$ & 0.936 \\
\hline
\end{tabular}

\section{Complications}

The postoperative complications are summarized in Table 7. There was no significant difference in complications related to low IOP such as shallow anterior chamber, choroidal detachment, and hypotony maculopathy. Since the Trab procedure requires the excision of the trabecular meshwork and the incision of the peripheral iris, the rates of vitreous hemorrhage were significantly higher in the Trab group $(p=0.047)$. 
Table 7

Complications

\begin{tabular}{|llll|}
\hline & Ex-press & Trab & p-value \\
\hline Shallow anterior chamber & 5 & 6 & 0.898 \\
\hline Choroidal detachment & 6 & 6 & 0.833 \\
\hline hypotony maculopathy & 0 & 0 & 1.000 \\
\hline Hyphema & 5 & 6 & 0.898 \\
\hline Tube occlusion & 0 & 0 & 1.000 \\
\hline Vitreous hemorrhage & 0 & 3 & 0.047 \\
\hline Vitreous prolapse & 0 & 2 & 0.256 \\
\hline Leaking bleb & 1 & 4 & 0.190 \\
\hline Visual acuity aggravation & 9 & 10 & 0.988 \\
\hline Bullous keratopathy & 1 & 1 & 0.936 \\
\hline
\end{tabular}

\section{Discussion}

The Ex-PRESS surgery and the trabeculectomy both significantly reduced IOP even in cases of low preoperative IOP. The trabeculectomy reduced IOP more compared to the EXP. There was no significant between-group difference in the surgeries' success rates.

There have been several reports that both EXP and Trab are useful for treating low-IOP glaucoma. Naito et al. reported that Trab lowered the IOP from 13.9 to $8.1 \mathrm{mmHg}$ at 2 years [4], and Schultz et al. stated that Trab lowered the IOP from 13.1 to $8.5 \mathrm{mmHg}$ [9]. The present surgical outcomes are equivalent to these previous reports, which also indicated that Trab could suppress the progression of visual field deterioration $[4,9]$.

In a study by Aihara et al., the use of EXP lowered the IOP from 14.8 to $10.0 \mathrm{mmHg}$ in one year, achieving a $31.1 \%$ reduction of IOP [15]. In our present study, the IOP reduction provided by EXP was equivalent to $28.0 \%$ at 1 year post-surgery. The Collaborative NTG study reported that a $30 \%$ reduction was recommended for normal-tension glaucoma (NTG) [19]. In our present patient series, the reduction in the EXP group at 2 years was $22.8 \%$, whereas that in the Trab group was $33.4 \%$. The reason for this difference is that in the EXP procedure, a small amount of aqueous humor can flow out, and as a result, the IOP might be higher than that achieved with a Trab. We reported that the volume of filtered blebs after EXP declined by $26 \%$ per year [20]. It is expected that a large bleb will be maintained with a low IOP for a long period. The trabeculectomy has the advantage of lowering the IOP to a greater degree compared to EXP. Trab had a double layer scleral flap, which could decrease IOP more. 
There are several ways to define success after glaucoma surgery [21]. Since the present patients' preoperative IOP was low, we did not use a cut-off IOP value for the definition of surgical success. There was no significant difference between the Trab and EXP surgeries when the $<20 \%$ or $<30 \%$ reductions in IOP were successful. Considering that a $30 \%$ reduction is recommended, Trab seems to be slightly better. This study was a retrospective analysis and did not compare preoperative and postoperative visual field results; it is thus unclear whether a $20 \%$ reduction in the IOP is sufficiently effective. Oie et al. reported that there was a correlation between the IOP reduction ratio and the speed of deterioration in the visual field [22].

We observe that the postoperative VA was less deteriorated in the EXP group. Since our study included cases of simultaneous cataract surgery, we could not investigate the changes in VA affected by glaucoma surgery without this factor. In the Trab group, the VA was more likely to deteriorate despite the inclusion of more patients with simultaneous cataract surgeries in this group. This might be have contributed to the decrease in the IOP being too low. Naito et al. reported that an IOP $<7 \mathrm{mmHg}$ posed a risk of a decline in the VA [4]. An investigation by Beltran et al. revealed that patients who underwent a Trab were more likely to lose $\geq 2$ Snellen lines compared to those who underwent an EXP [23].

Astigmatism is likely to occur when the IOP is low [24], and astigmatism might affect VA. Several studies reported that VA recovery after EXP was more rapid than that after Trab [18, 23, 25]. EXP might therefore have an advantage concerning postoperative VA.

In the present study, there was no significant between-group difference in the reduction ratio of ECD at 2 years post-surgery, but the reduction of ECD was slightly greater in the Trab group. Several studies reported that Trab or EXP reduced the ECD by $2.2-23.0 \%$ in a 2-year period [13, 17, 26, 27]; however, in one of the studies the EXP surgery reduced the ECD rapidly [13], whereas others observed that EXP could not reduce ECD rapidly $[17,28]$. We reported that postoperative ECD varies depending on the insertion position of the EXP [29]. If the EXP is inserted in the correct position (the trabecular meshwork), it might prevent ECD loss more effectively. The EXP procedure does not require the resection of the iris and trabecular meshwork, and it results in less inflammation compared to a Trab. Strong inflammation might decrease the ECD more rapidly [30,31].

There are many reports that EXP has fewer complications than Trab $[11,17,18]$. In the present study as well, the vitreous hemorrhage and vitreous prolapse that are characteristic of a Trab did not occur in the EXP group. For these reasons, EXP might pose a lower risk of complications. The EXP also has a characteristic complication of tube obstruction [32], but this did not occur in the present series of patients.

The preoperative IOPs of our patients were very low, and surgeries that require further IOP reduction could pose a high risk of complications associated with low IOP such as shallow anterior chamber, choroidal detachment, and hypotony maculopathy. Notably, the Trab results in more outflow to outside of the eye, and we thus suspected that the Trab might result in more complications associated with low IOP. However, there was no between-group difference in complications associated with low IOP. Arimura et al. 
reported that EXP caused choroidal detachment in $18 \%$ of their patients whereas Trab caused it in $12.5 \%$, which was not a significant difference [17]. Our present results are similar. Appropriate laser suture-lysis could lower the risk of complications.

In terms of medical economy, Patel et al. reported that EXP was associated with greater surgical cost compared with Trab [33].

There are some study limitations to address. This was a retrospective analysis. There is a risk that the results would vary greatly depending on the preoperative IOP values. We did not consider IOP fluctuations. Our patient population included cases with simultaneous cataract surgery, and it has been reported that simultaneous cataract surgery has poorer surgical results and is more likely to reduce the ECD rapidly $[34,35]$. We did not define the indications for glaucoma surgery, cataract surgery, and additional glaucoma medications. The number of patients was small, and the follow-up period was short.

Even in an era when many glaucoma devices are available, the number of surgical methods for patients with low preoperative IOP might be limited. Since both EXP and Trab could adjust the postoperative IOP to some extent, these surgeries were considered first for our patients. Trab has a greater ability to lower the IOP. Since the surgical results of glaucoma focus on the postoperative IOP, it seems that Trab is the better surgical method. However, we cannot recommend surgery that has a high possibility of postoperative complications, even if the IOP decreases more. Both surgeries were useful for patients with low preoperative IOP, and both have advantages and disadvantages. It is necessary to judge these surgeries by the outcomes over a long-term follow-up.

\section{Declarations}

Funding statement: No funding was received for this research.

Financial interests and conflict of Interest statement: The authors have no relevant financial or nonfinancial interests to disclose.

Author Contributions: All authors contributed to the study conception and design. Material preparation, data collection and analysis were performed by Mitsuya Otsuka and Naoki Tojo. The first draft of the manuscript was written by Mitsuya Otsuka and all authors commented on previous versions of the manuscript. All authors read and approved the final manuscript.

Ethical approval: All procedures were performed in accord with the ethical standards of the Institutional Review Board of the University of Toyama (Toyama, Japan) and the 1964 Helsinki Declaration and its later amendments or comparable ethical standards. Formal patient consent was not required for the present retrospective analysis.

Consent to participate and publish: Informed consent was obtained from all individual participants included in the study. 


\section{References}

1. Lavia C, Dallorto L, Maule M, Ceccarelli M, Fea AM (2017) Minimally-invasive glaucoma surgeries (MIGS) for open angle glaucoma: A systematic review and meta-analysis. PLOS ONE. 12(8): 0183142.

2. Coleman AL, Hill R, Wilson MR, Choplin N, Kotas-Neumann R, Tam M, et al. (1995) Initial clinical experience with the Ahmed Glaucoma Valve implant. Am J Ophthalmol. 120(1):23-31.

3. Iwase A, Suzuki Y, Araie M, Yamamoto T, Abe H, Shirato S, et al. (2004) The prevalence of primary open-angle glaucoma in Japanese: The Tajimi Study. Ophthalmology. 111(9):1641-8.

4. Naito T, Fujiwara M, Miki T, Araki R, Fujiwara A, Shiode Y, et al. (2017) Effect of trabeculectomy on visual field progression in Japanese progressive normal-tension glaucoma with intraocular pressure $<15$ mmHg. PLOS ONE. 12(8):e0184096.

5. Aoyama A, Ishida K, Sawada A, Yamamoto T (2010) Target intraocular pressure for stability of visual field loss progression in normal-tension glaucoma. Jpn J Ophthalmol. 54(2):117-23.

6. Iverson SM, Schultz SK, Shi W, Feuer WJ, Greenfield DS (2016) Effectiveness of single-digit IOP targets on decreasing global and localized visual vield progression after fltration surgery in eyes with progressive normal-tension glaucoma. J Glaucoma. 25(5):408-14.

7. Yuasa Y, Sugimoto Y, Hirooka K, Ohkubo S, Higashide T, Sugiyama K, et al. (2020) Effectiveness of trabeculectomy with mitomycin $\mathrm{C}$ for glaucomatous eyes with low intraocular pressure on treatment eye drops. Acta Ophthalmol 98(1):e81-e7.

8. Nakajima K, Sakata R, Ueda K, Fujita A, Fujishiro T, Honjo M, et al. (2021) Central visual field change after fornix-based trabeculectomy in Japanese normal-tension glaucoma patients managed under $15 \mathrm{mmHg}$. Graefes Arch Clin Exp Ophthalmol. 259(8):2309-16.

9. Schultz SK, Iverson SM, Shi W, Greenfield DS (2016) Safety and efficacy of achieving single-digit intraocular pressure targets with filtration surgery in eyes with progressive normal-tension glaucoma. J Glaucoma. 25(2):217-22.

10. Wamsley S, Moster MR, Rai S, Alvim HS, Fontanarosa J (2004) Results of the use of the Ex-PRESS miniature glaucoma implant in technically challenging, advanced glaucoma cases: A clinical pilot study. Am J Ophthalmol. 138(6):1049-51.

11. Wang L, Sha F, Guo DD, Bi HS, Si JK, Du YX, et al (2016) Efficacy and economic analysis of Ex-PRESS implantation versus trabeculectomy in uncontrolled glaucoma: A systematic review and Metaanalysis. Int J Ophthalmol. 9(1):124-31.

12. Nicolai M, Franceschi A, Pelliccioni P, Pirani V, Mariotti C (2020) EX-PRESS glaucoma filtration device: Management of complications. Vision. 4(3).

13. Ishida K, Moroto N, Murata K, Yamamoto T (2017) Effect of glaucoma implant surgery on intraocular pressure reduction, flare count, anterior chamber depth, and corneal endothelium in primary openangle glaucoma. Jpn J Ophthalmol. 61(4):334-46. 
14. Kato N, Takahashi G, Kumegawa K, Kabata Y, Tsuneoka H (2015) Indications and postoperative treatment for Ex-PRESS $®$ insertion in Japanese patients with glaucoma: comparison with standard trabeculectomy. Clin Ophthalmol. 9:1491-8.

15. Aihara M, Kuwayama Y, Miyata K, Ohtani S, Ideta R, Hashimoto Y, et al. (2019) Twelve-month efficacy and safety of glaucoma filtration device for surgery in patients with normal-tension glaucoma. Jpn J Ophthalmol. 63(5):402-9.

16. Wagschal LD, Trope GE, Jinapriya D, Jin YP, Buys YM (2015) Prospective randomized study comparing Ex-PRESS to trabeculectomy: 1-Year results. J Glaucoma. 24(8):624-9.

17. Arimura S, Takihara Y, Miyake S, Iwasaki K, Gozawa M, Matsumura T, et al. (2016) Randomized clinical trial for early postoperative complications of Ex-PRESS implantation versus trabeculectomy: Complications Postoperatively of Ex-PRESS versus Trabeculectomy Study (CPETS). Sci Rep. 6:26080.

18. Netland PA, Sarkisian SR, Jr., Moster MR, Ahmed, II, Condon G, Salim S, et al. (2014) Randomized, prospective, comparative trial of EX-PRESS glaucoma filtration device versus trabeculectomy (XVT study). Am J Ophthalmol. 157(2):433-40 e3.

19. No authors listed (1998) The effectiveness of intraocular pressure reduction in the treatment of normal-tension glaucoma. Collaborative Normal-Tension Glaucoma Study Group. Am J Ophthalmol. 126(4):498-505.

20. Tojo N, Hayashi A, Otsuka M (2018) Factors influencing the filtration-bleb volume after Ex-PRESS ${ }^{\circledR}$ surgery. Clin Ophthalmol. 12:1675-83.

21. Rotchford AP, King AJ (2010) Moving the goal posts definitions of success after glaucoma surgery and their effect on reported outcome. Ophthalmology. 117(1):18-23 e3.

22. Oie S, Ishida K, Yamamoto T (2017) Impact of intraocular pressure reduction on visual field progression in normal-tension glaucoma followed up over 15 years. Jpn J Ophthalmol. 61(4):314-23.

23. Beltran-Agullo L, Trope GE, Jin Y, Wagschal LD, Jinapriya D, Buys YM (2015) Comparison of visual recovery following ex-PRESS versus trabeculectomy: Results of a prospective randomized controlled trial. J Glaucoma. 24(3):181-6.

24. Tanito M, Matsuzaki Y, Ikeda Y, Fujihara E (2017) Comparison of surgically induced astigmatism following different glaucoma operations. Clin Ophthalmol. 11:2113-20.

25. Kobayashi N, Hirooka K, Nitta E, Ukegawa K, Tsujikawa A (2018) Visual acuity and corneal higherorder aberrations after EX-PRESS or trabeculectomy, and the determination of associated factors that influence visual function. Int Ophthalmol. 38(5):1969-76.

26. Higashide T, Nishino T, Sakaguchi K, Yamada Y, Sugiyama K (2019) Determinants of corneal endothelial cell loss after trabeculectomy with mitomycin C. J Glaucoma. 28(1):61-7.

27. Omatsu S, Hirooka K, Nitta E, Ukegawa K (2018) Changes in corneal endothelial cells after trabeculectomy and EX-PRESS shunt: 2-year follow-up. BMC Ophthalmol. 18(1):243.

28. Hirooka K, Nitta E, Ukegawa K, Sato S, Kiuchi Y (2020) Effect of trabeculectomy on corneal endothelial cell loss. Br J Ophthalmol. 104(3):376-80. 
29. Tojo N, Numata A, Hayashi A (2020) Factors influencing the reduction in corneal endothelial cells after Ex-Press ${ }^{\circledR}$ surgery. Int Ophthalmol. 40(5):1201-8.

30. Smith DL, Skuta GL, Lindenmuth KA, Musch DC, Bergstrom TJ (1991) The effect of glaucoma filtering surgery on corneal endothelial cell density. Ophthalmic Surg. 22(5):251-5.

31. Macdonald JM, Geroski DH, Edelhauser HF (1987) Effect of inflammation on the corneal endothelial pump and barrier. Curr Eye Res. 6(9):1125-32.

32. Chan JE, Netland PA (2015) EX-PRESS Glaucoma Filtration Device: Efficacy, safety, and predictability. Med Devices. 8:381-8.

33. Patel HY, Wagschal LD, Trope GE, Buys YM (2014) Economic analysis of the Ex-PRESS miniature glaucoma device versus trabeculectomy. J Glaucoma. Aug;23(6):385-90.

34. Soro-Martinez MI, Villegas-Perez MP, Sobrado-Calvo P, Ruiz-Gomez JM, Miralles de Imperial MoraFigueroa J (2010) Corneal endothelial cell loss after trabeculectomy or after phacoemulsification, IOL implantation and trabeculectomy in 1 or 2 steps. Graefes Arch Clin Exp Ophthalmol. 248(2):24956.

35. Graffi S, Tiosano B, Naftali M, Nakhoul N, Mimouni M, Hanna R, et al (2020) Short-term anterior chamber inflammation in phacoemulsification with and without Ex-Press glaucoma implant. Eur $\mathrm{J}$ Ophthamol. 30(3):533-7.

\section{Figures}

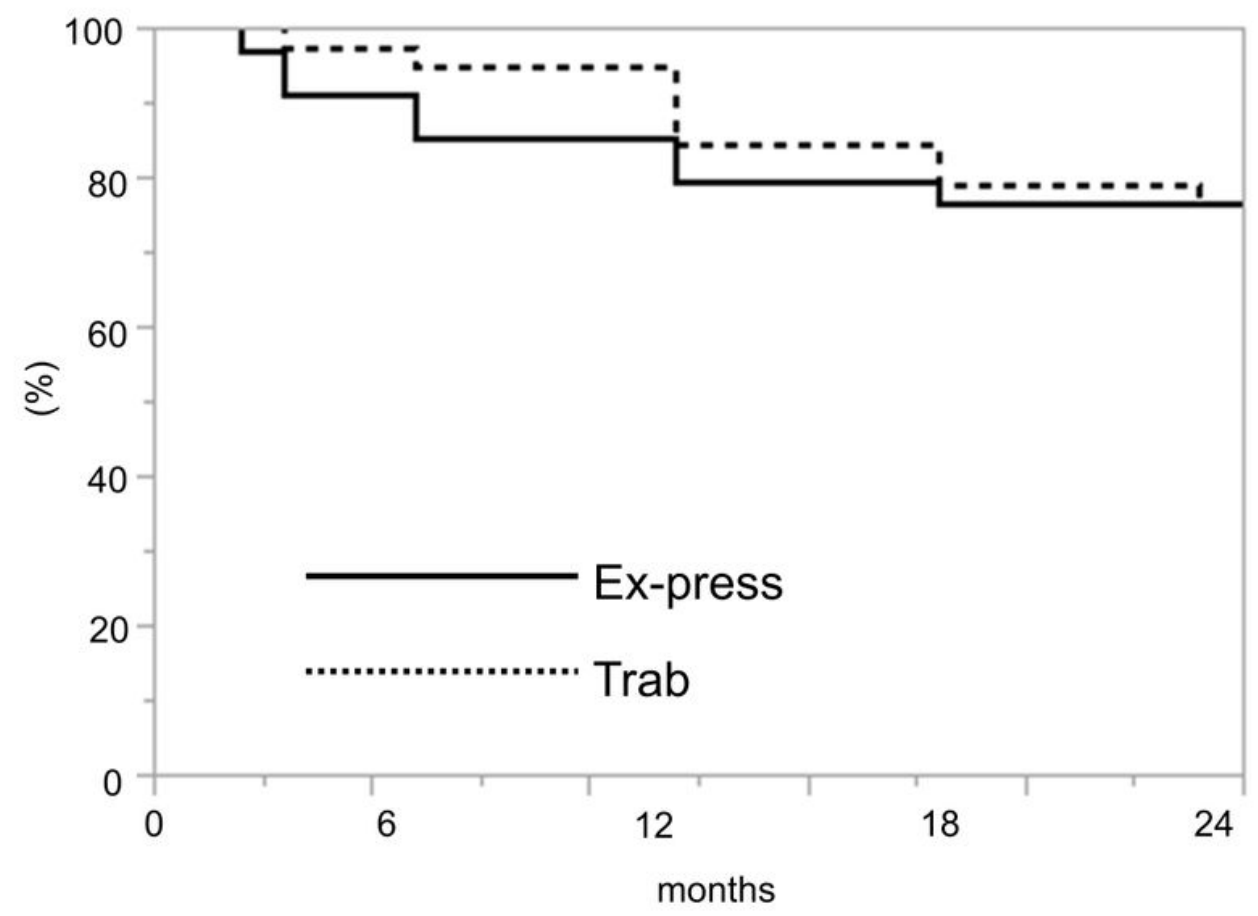

Fig. 1

Figure 1 
Caption not included with this version.

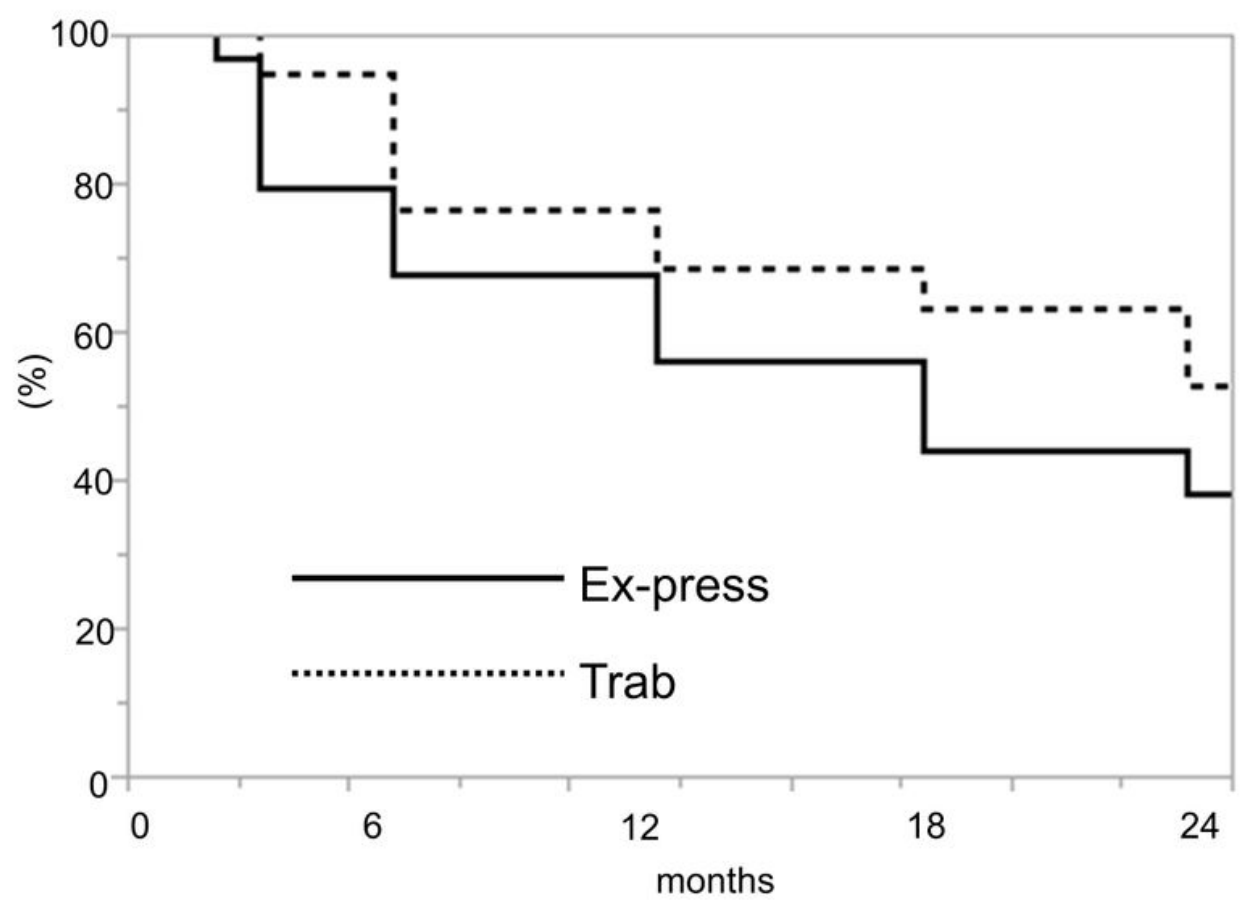

Fig. 2

Figure 2

Caption not included with this version. 